\title{
New approach allows derivation of microglia-like cells in vitro
}

Using a novel approach, researchers have derived microglia-like cells from human pluripotent stem cells for the first time, according to a recent report in Nature Medicine. The cells exhibited molecular signatures similar to those of microglia, and behaved like microglia in 3D neuroglial cultures, suggesting that the system will facilitate in vitro studies of neurological diseases.

Rudolf Jaenisch and colleagues, who conducted the study, have previously used human induced pluripotent stem cells (iPSCs) to model Parkinson disease, Rett syndrome and Niemann-Pick disease, and

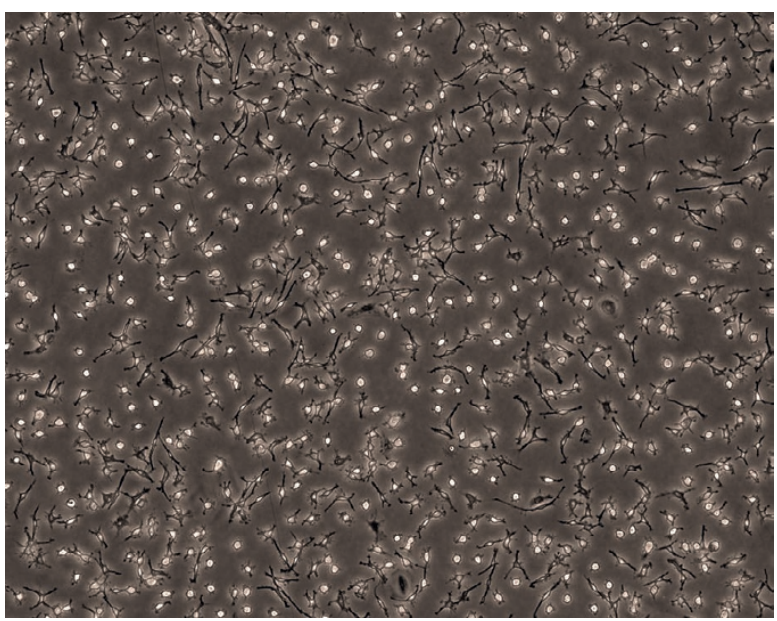

A lawn of microglia-like cells derived from human pluripotent stem cells. Image courtesy of J. Muffat. are currently modelling Alzheimer disease and adrenoleukodystrophy. However, they have been unable to include microglia in their models. "In all these diseases, the role of microglia is becoming more prominent every day, yet there were no good culture systems for primary human microglia, or clear differentiation protocols from iPSCs," explains Julien Muffat, the co-lead author of the study.

The researchers started by allowing spontaneous differentiation of human embryonic stem cells and iPSCs in a medium developed to mimic conditions in the CNS. They then grew populations of these cells in the presence of IL-34 and colonystimulating factor 1 , two factors that are important for differentiation and maintenance of microglia.

The resulting cells were positive for PU.1, CD11B and allograft inflammatory factor 1 , all of which are markers of microglia, and were also phagocytic and highly motile. The same procedure reliably produced such microglia-like cells from 20 different lines of pluripotent cells.

"These cells can be further 'neuralized' by exposure to conditioned media from differentiating neural progenitors," explains Muffat. "They can be maintained in culture for months as they mature further, and they recapitulate much of the signature of primary fetal microglia."

Muffat and colleagues integrated their microglia-like cells into 3D cultures of neurons and astrocytes to recapitulate the cellular environment in the CNS. In this setting, the cells took on a more complex, branched morphology characteristic of microglia in the human brain, and a laser-induced focal injury caused nearby microglia-like cells to extend processes and subsequently migrate to encapsulate the injury site.

"Our observations line up nicely with the current, yet evolving, knowledge about microglia, their origin, identity and function," says Muffat. "However, the cells we generated resemble fetal human microglia, and we want to show that the cells embedded in 3D culture adopt a more mature signature resembling postnatal — perhaps adult — human microglia."

The team hope that their novel platform will aid studies of disease and ageing by more accurately reproducing physiological conditions. "One can imagine pairing this platform with another 3D culture system developed by another group: Alzheimer-in-a-dish," says Muffat. "In this context, one can expect to observe the interactions of microglia with amyloid plaques or dystrophic neurites, leading to better opportunities for drug discovery."

ORIGINAL ARTICLE Muffat, J. et al. Efficient derivation of microglia-like cells from human pluripotent stem cells. Nat. Med. http://dx.doi. org/10.1038/nm.4189 (2016) 